\title{
Stress Concentration Minimization of 2D filets using X-FEM and Level Set Description
}

\begin{abstract}
This paper presents and applies a novel shape optimization approach based on the Level Set description of the geometry and the eXtended Finite Element Method (XFEM). The method benefits from the fixed mesh work using X-FEM and from the curves smoothness of the Level Set description. Design variables are shape parameters of basic geometric features which are described with a Level Set representation. The number of design variables of this formulation remains small whereas global (i.e. compliance) and local constraints (i.e. stress) can be considered. To illustrate the capability of the method to handle stress constraints, numerical applications revisit the minimization of stress concentration in a 2D filet in tension which has been studied previously by Pedersen in (27). Our results illustrate the great interest of using X-FEM and Level Set description together. A special attention is also paid to stress computation and accuracy with the X-FEM.
\end{abstract}

Keywords Shape optimization - Topology optimization · $\mathrm{X}$-FEM $\cdot$ Level set

\section{Introduction}

Minimizing stress concentrations has been the topics of many works in structural optimization. Reducing stress concentrations and stress intensity factors or controlling the stress failure criteria is essential in mechanical engineering design (see for instance Ref. (29)). Very early structural

P. Duysin $\mathrm{x}$

Automotive Engineering

Aerospace and Mechanics Department - University of Liege

Chemin des Chevreuils, 1, building B52, 4000 Liege, Belgium

Tel.: +32-43669194

Fax: +32-43669159

E-mail: P.Duysinx@ulg.ac.be

L. Van Miegroet

Automotive Engineering

Aerospace and Mechanics Department - University of Liege

Chemin des Chevreuils, 1, building B52, 4000 Liege, Belgium

Tel.: +32-43669270

Fax: +32-43669159

E-mail: L.VanMiegroet@ulg.ac.be optimization techniques have been identified as a powerful and rationale tool to support the engineering task in finding designs with high stiffness and strength. While sizing variables bring a rather limited improvement of stress concentration factors, shape optimization can substantially increase the strength of the design by modifying the boundaries of the structures. The reader can refer to the two famous review papers by Ding (12) or Haftka and Grandhi (17) and the references cited therein. Among many papers one can mention the contributions by Bennet and Botkin (7), Braibant and Fleury (8), Pedersen and Laursen (24), which proposed several applications of shape optimization to reduce stress concentrations and failure criteria.

Following the impetus initiated by the work of Bendsøe and Kikuchi (3), a very important amount of research has focused on topology optimization. As topology optimization is formulated as an optimal material distribution, topology optimization introduces a very large number of design variables so that most of the works consider only global or integral objective function with a single volume constraint. Due to the large number of restrictions to be considered, works including stress constraints are rather limited (see for instance Duysinx and Bendsøe, (13) and Pereira et al. (28), Allaire et al. (2)). Fortunately for a single load case, Pedersen in Ref. (26) showed that compliance minimization leads to a good improvement of stress concentration. However for multiple load case, unequal stress constraints or multi material problems it is known (see for instance Rozvany (30)) that there is no guarantee that optimum designs for stiffness and strength are the same and the stress constraints have to be introduced in the design problem formulation.

While topology was experiencing a very spectacular success in industrial applications, shape optimization has been more difficult to apply in industrial environment. Practically, one major advantage of the optimal material distribution formulation is to be able to work on a fixed regular mesh, while shape optimization is forced to struggle with difficult problems related to mesh distorsion, automatic mesh regeneration problems, finite element errors, etc. Moreover a major technical problem also stems from the sensitivity analysis that requires the calculation of the so-called velocity field 
because of the moving mesh and it affects the complexity and cost of the sensitivity analysis. It turns out that shape optimization remains generally quite fragile and delicate to use in industrial context.

In order to circumvent the technical difficulties of the moving mesh problems, a couple of researches have tried to apply the fixed mesh principle to shape optimization. Let's mention the use of fictitious domain approach by Haslinger and co-authors as in Ref. (10), the fixed grid finite elements by Kim et al. in Ref. (18) or more recently the projection methods as in Norato et al. (20). The present work relies on the novel eXtended Finite Element Method (X-FEM) that has been proposed as an alternative to remeshing methods (see Ref. (19) or (5) for instance). The X-FEM method is naturally associated with the Level Set proposed by Osher and Sethian (Ref. (23)) description of the geometry to provide a very efficient treatment of difficult problems involving discontinuities and propagations. Up to now the X-FEM method has been mostly developed for crack propagation problems (19), but the potential interest of the X-FEM and the Level Set description for other problems like topology optimization was identified very early in Belytschko et al. (6), while the advantages of the Level Set method in structural optimization was clearly demonstrated by Wang et al. (34) or Allaire et al. (1).

In Ref. (14), the authors see the X-FEM and the Level Set description as an elegant way to fill the gap between topology and shape optimization and the method can be qualified as generalized shape optimization as it is based on smooth boundary descriptions while allowing topology modifications as holes can merge and disappear. X-FEM enables working on a fixed mesh, as in topology optimization, removing the technical difficulties related to the mesh and velocity field management from the formulation. The optimization formulation has the advantage of using a small number of design variables related to the shape parameters of geometric model of the structure described in Level Sets entities, while constraints can be either global (compliance, volume) or local (stresses) responses as in shape optimization.

The work wants to demonstrate that stress constraints can be considered efficiently when using X-FEM and not only compliance or energy functions. To this end the paper illustrates the capabilities of the methodology to minimize stress concentration factors in a well known family of benchmarks coming from shape optimization. The example of 2D filets has been selected because of the numerous published results for this test case.

The layout of the paper is organized as follows. At first in section 2 the Extended Finite Element Method is presented for cracks, bi material and void. A special attention is also paid to stress computation and accuracy with the X-FEM. Then, section 3 summarizes the Level Set representation and its combination with X-FEM. In section 4 the formulation of optimization problem is presented whereas the sensitivity analysis is addressed in section 5. Finally in section 6, 2D filet stress concentration minimization problems (inspired by Ref. (27)) are used to illustrate optimization strategy using X-FEM and Level Set when stress constraints are considered.

\section{The eXtended Finite Element Method}

The eXtended Finite Element Method (X-FEM) (19; 5) has been firstly introduced to study propagating cracks in mechanical structures. The method has obtained so promising results in fracture mechanics that some authors have immediately foreseen the opportunities of applying X-FEM to many kinds of problems in which discontinuities and moving boundaries have to be modeled. As exemple publications we can mention Belytschko et al. (5) who applied the XFEM to the modelization of composite fibre orientation in a micro-structure, Chessa et al. (9) who studied the case of two phase fluid problems, and recently Guetari et al. (16) who simulated material removal in machine tooling problems using X-FEM formalism.

\subsection{Principle of the method}

In the classical Finite Element Method, modeling discontinuities inside an element is not possible because the shape functions are required to be at least $C^{1}$. Therefore, if discontinuities are present in the model, these discontinuity boundaries have to coincide with the element mesh boundaries and they require a remeshing process each time the singularity evolves in the structure.

The X-FEM overcomes this restriction by adding to the classical FEM approximation some particular discontinuous or singular shape functions. Hence, these additional shape functions, directly related to the nature of the discontinuity, allow to include geometric boundaries, cracks, material or phase changes that are not coincident with the mesh and avoid the expensive and delicate mesh generation.

\subsection{Modeling cracks}

In presence of cracked structures, the displacement is discontinuous. Hence, modeling the displacement field calls for discontinuous shape functions. The classical finite element approximation used is then extended to embed the discontinuous shape function as in the following equation:

$\mathbf{u}(\mathbf{x})=\sum_{i} u_{i} N_{i}(\mathbf{x})+\sum_{j} a_{j} N_{j}(\mathbf{x}) H(\mathbf{x})$

where $N_{i}(\mathbf{x})$ are the classical shape functions related to nodal degrees of freedom $u_{i}$. The $N_{j}(\mathbf{x}) H(\mathbf{x})$ are the discontinuous shape functions constructed by multiplying a classical shape function $N_{j}(\mathbf{x})$ with a Heaviside function $H(\mathbf{x})$, which is equal to +1 on one side of the crack and -1 on the other side (see Fig. 1) . 


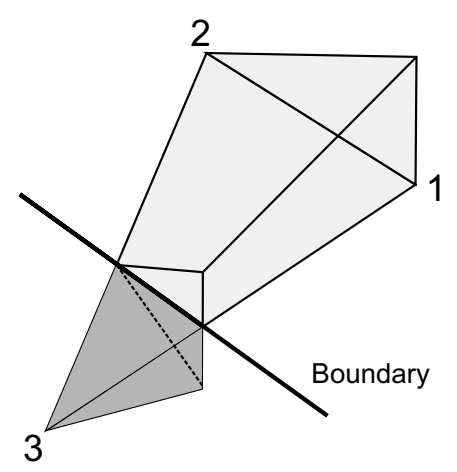

Fig. 1 Representation of the extended shape function $N_{1}(\mathbf{x}) H(\mathbf{x})$ of node 1 for a cracked element

Note that this set of extended shape functions are only supported by the enrichment degrees of freedom $a_{j}$. Moreover, only the elements near the discontinuity usually support extended shape functions whereas the other elements remain unchanged (i.e. classical FE). The modification of the displacement field approximation does not introduce a new form of the discretized finite element equilibrium equation but rather leads to an enlarged problem (see Ref. (19) for details):

$\mathbf{K} \cdot \mathbf{q}=\mathbf{g} \Leftrightarrow\left[\begin{array}{ll}K_{u u} & K_{u a} \\ K_{a u} & K_{a a}\end{array}\right]\left[\begin{array}{l}u \\ a\end{array}\right]=\left[\begin{array}{l}f_{u}^{e x t} \\ f_{a}^{e x t}\end{array}\right]$

As the elements can now present discontinuous shape functions, the numerical integration scheme has to be modified in order to take care of the discontinuity. The implementation used here is similar to the one described in Ref. (4). In order to use the classical Gauss numerical integration schemes, the elements embedding a singularity are divided into subtriangular mesh coincident with the discontinuity boundary. Over this working mesh, a quadrature integration rule can be applied (see Fig. 9). The procedure is described in more details in section 2.5 .

\subsection{Modeling material-material interfaces}

When an interface between two different materials is present, the displacement field is continuous but the strain field is discontinuous. Therefore, in order to model this kind of discontinuity inside an element, one have to add special shape functions that contain a discontinuity of the derivative of the displacement field. According to Ref. (9) and (31), one can use the following expression to discretize the displacement field:

$\mathbf{u}(\mathbf{x})=\sum_{i} u_{i} N_{i}(\mathbf{x})+\sum_{j} a_{j} N_{j}(\mathbf{x})\left(\left|\phi_{j}\right|-|\phi(\mathbf{x})|\right)$

where $\phi_{j}$ is the distance from the node $j$ to the interface and $\phi(\mathbf{x})$ is the distance of the current point $\mathbf{x}$ to the interface.

In order to illustrate this approximation, let's consider the simple problem of a bimaterial beam of Young modulus $E_{1}=1$ and $E_{2}=2$ subject to a traction force $F=1$ along the

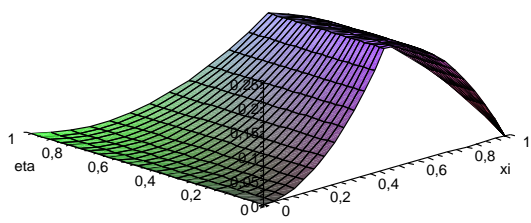

Fig. 2 Representation of the shape function of node 1 for a bi-material triangular element

$x$ axis (Fig. 3). With classical finite elements approximation, this very simple test case requires a minimum of two finite elements to be modeled. Within the X-FEM framework, the analysis of the problem can be carried out with a single $\mathrm{X}$ FEM. Using the enrichment of the shape functions proposed in eq. (3), the axial displacement field of the X-FEM rod is:

$$
\begin{aligned}
\mathbf{u}(\mathbf{x})= & u_{1}\left(1-\frac{\mathbf{x}}{L}\right)+u_{2}\left(\frac{\mathbf{x}}{L}\right)+a_{1}\left(1-\frac{\mathbf{x}}{L}\right) \mathbf{x}+a_{2}\left(\frac{\mathbf{x}}{L}\right) \mathbf{x}, \\
& \quad \mathbf{x} \in[0, L / 2] \\
\mathbf{u}(\mathbf{x})= & u_{1}\left(1-\frac{\mathbf{x}}{L}\right)+u_{2}\left(\frac{\mathbf{x}}{L}\right)+a_{1}\left(1-\frac{\mathbf{x}}{L}\right)(L-\mathbf{x}) \\
+ & a_{2}\left(\frac{\mathbf{x}}{L}\right)(L-\mathbf{x}), \mathbf{x} \in[L / 2, L]
\end{aligned}
$$

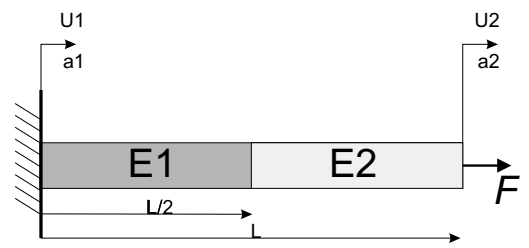

Fig. 3 Geometrical model

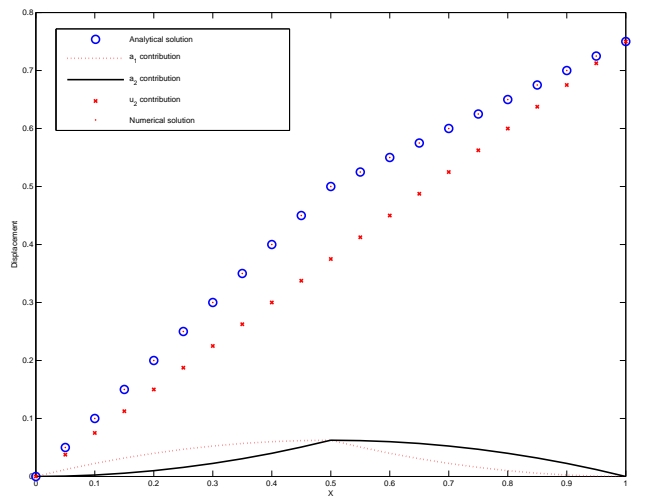

Fig. 4 Degrees of freeedom contributions, analytical and numerical solutions 
Different enrichment shape functions can be used to model a bimaterial interface (see Ref. (31) for more details), however, the displacement field of eqn. (4) and (5) has the particular and attractive advantage of zeroing on the two end nodes, which prevent from connecting the degree of freedom $a_{j}$ to other elements.

\subsection{Modeling material-void interfaces}

The modeling of material-void interfaces with X-FEM (31) differs only marginally from the cracked structure case (i.e. the discontinuous field is the displacement). For void inclusions and holes, the displacement field is now approximated by:

$\mathbf{u}(\mathbf{x})=\sum_{i} u_{i} N_{i}(\mathbf{x}) V(\mathbf{x})$

where

$V(\mathbf{x})=\left\{\begin{array}{lll}0 & \text { if } & \mathbf{x} \in \text { material zone } \\ 1 \text { if } & \mathbf{x} \in \text { void }\end{array}\right.$

One can note that this function $V(\mathbf{x})$ models perfectly the singularity in the displacement field as the function $V(\mathbf{x})$ cancels the displacement field in the void (see Fig. 5).



Fig. 5 Representation of the shape function of node 1 on a cut element

Modeling holes with the X-FEM is a very appealing method for the shape optimization but also for the topology optimization as no remeshing is needed and no approximation is made on the nature of the voids conversely to numerous methods as the power penalization of intermediate densities method used (SIMP) in which void is replaced by a very weak material. However, as we will note in section 2.5 , the X-FEM method requires more complicated integration procedure and a careful attention has to be paid to stress and sensitivity computation.

\subsection{Implementation of X-FEM}

In the case of material-void interfaces, elements are of only three types: solid ones, void ones and cut ones. The void elements have all their nodes lying fully in the void. These elements (red ones in Fig. 6) are then consequently removed from the problem and have no contribution to the stiffness matrix. Their nodal degrees of freedom are eliminated from the system of equations when assembling the global stiffness matrix. The filled elements (green ones in Fig. 6) with all nodes lying in the solid domain are treated as normal elements whereas the partially filled elements (blue ones in Fig. 6 ), which have a mix of void and solid nodes require purely an X-FEM.



Fig. 6 Representation of the different element type

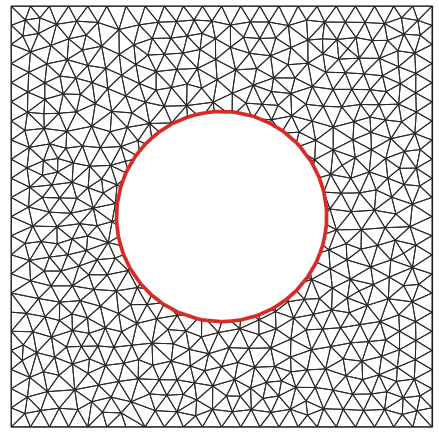

Fig. 7 Representation of the model with cut and filled element only

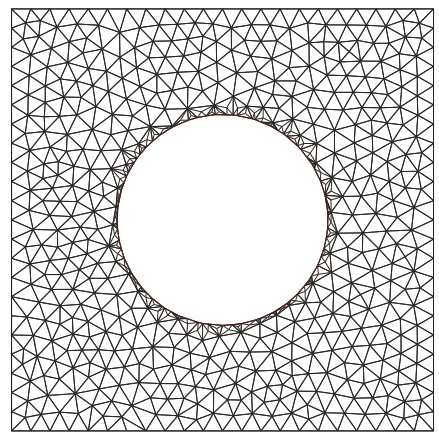

Fig. 8 Representation of the model with the sub-triangles 


\subsubsection{Numerical integration of the stiffness matrix}

First of all when an X-FEM element is to be integrated, we first have to detect which part of the element is filled with material in order to determine if the solid part is a triangle or a quadrangle. As one can see in section 3 devoted to the Level Set description of the geometry, this is easily done when using a Level Set Description.

For X-FEM elements a major issue is the computation of the stiffness matrix. Because of the strong discontinuity of the shape functions that takes place at the void solid interface, one can observe that using a quadrature rule (e.g. Gauss points) would lead to a totally unsatisfactory results even when increasing dramatically the number of Gauss points. At first one has to notice that because of the zero displacement field in the void domain (see eqn. 6), the void part of the element does not contribute to the stiffness matrix. Thus the computation is restricted to an integration procedure solely over the solid sub-domain of the element(see Fig. 8 ). To integrate over the solid part of the element domain, we divide it into a partition of triangles in 2D (or tetrahedrons in 3D) and use a classical Gauss quadrature over each of the sub-triangles. As the field is linear, Gauss quadrature rule is exact on each sub-triangles. The procedure can easily be developed either for triangular or quadrangular sub-domains. The main idea is to use the center of gravity to support a new integration mesh. The Fig. 9 illustrates the domain subdivision of a cut element in order to integrate it. One has to notice that the sub-triangles are only a background mesh only used for the integration and that the size of the problem is not augmented regarding to a classical finite element model.

Attention has still to be paid to two kinds of numerical problems that can occur with the X-FEM modeling of void. The first one happens when the remaining solid part of the element is a quadrangle that is nearly degenerated into a triangle. The case is illustrated in Fig. 10. Hence, this quadrangle could be divided into four triangles, but one of these triangles would be very ill conditioned and would introduce a rank deficiency. Thus, it is better to consider this solid domain of the element as a triangle and divide it into three triangles. The error is very limited and the precision is good, which would never be achieved with a degenerated sub-triangle .

The second kind of numerical error occurs when the area of the solid part becomes very small regarding to the area of the supporting element (Fig. 11). One can observe that in this case the element becomes very stiff and the structural stiffness matrix becomes somewhat not so well conditioned. This explains the reason why some authors (for example see Ref. (11)) do not include these elements and eliminate them from the model when building the stiffness matrix. In this study we could circumvent the problem thanks to a scaling procedure of the stiffness matrix and we could not notice any degradation of the precision in the displacement field.

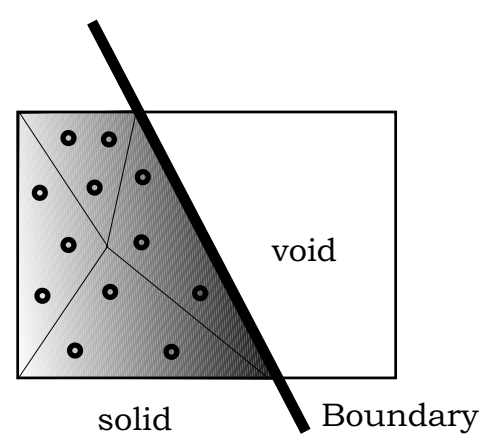

(a) Sub-division of a quadrangular element and Gauss points

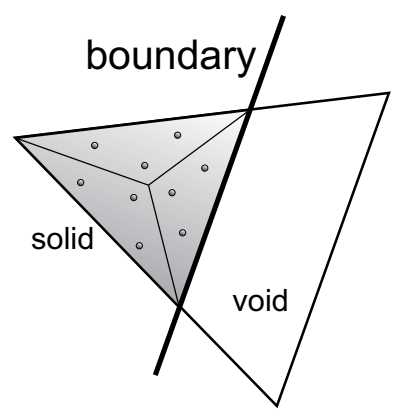

(b) Sub-division of a quadrangular element and Gauss points

Fig. 9 Sub division of elements

Especially compliance value has always been in very good agreement with FEM that is taken as a reference solution as shown in Ref. (32). However we could observe that these very small solid elements are the source of a more important difficulty resulting in a very high overestimation of the local stress. This is a crucial issue in this study in which we want to minimize the stress level. The problem is explained in the next section.



Fig. 10 Quadrangle degenerated into a triangle

\subsubsection{Estimation of stresses}

A test case illustrates the problem of the overestimation of the stress when the area of the solid part becomes very small 


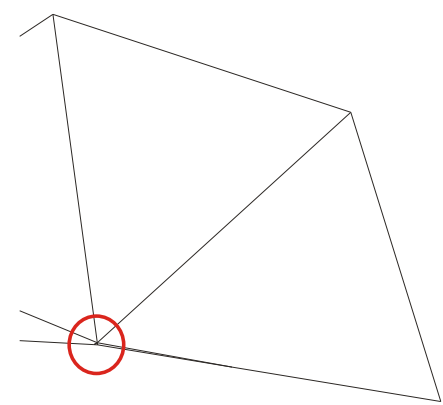

Fig. 11 Small solid triangle

regarding to the area of the supporting element. The problem is a classical quarter plate with a hole under an uniaxial load as illustrated in Fig. 12 and Fig. 13. The Fig. 12 shows the stress component along the $x$ (computed at Gauss point) obtained with a FEM model, whereas the second Fig. 13. illustrates the same result given by an X-FEM analysis.

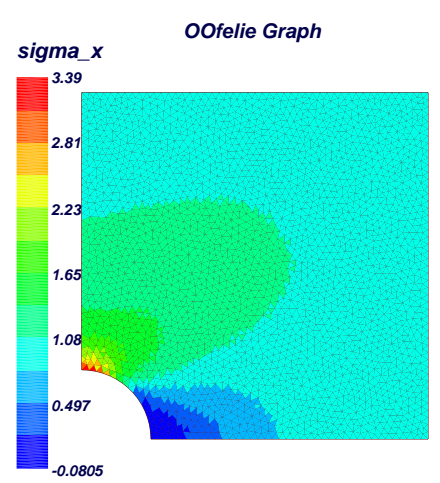

Fig. 12 FEM stress distribution

As we can remark, a large error occurs on the maximum stress for the X-FEM model. This overestimation of the stress level is due to a small solid element as pointed out in the zoomed part of Fig. 13. This is even more clear when we consider the following experiment. If the boundary of the circle is slightly modified (perturbation of $10^{-4}$ of the radius circle) in order to remove the small cut element, it is seen that the agreement between the results obtained with the two methods become excellent again.

To prevent the phenomenon, one can resort to the following strategy:

- Eliminate elements that have a too small solid part from the model when building the stiffness matrix (see (11));

- Move the node on the boundary in order to remove the small elements;

- Post process the stress results and eliminate stresses when the ratio of the solid area to the supporting area is too low;

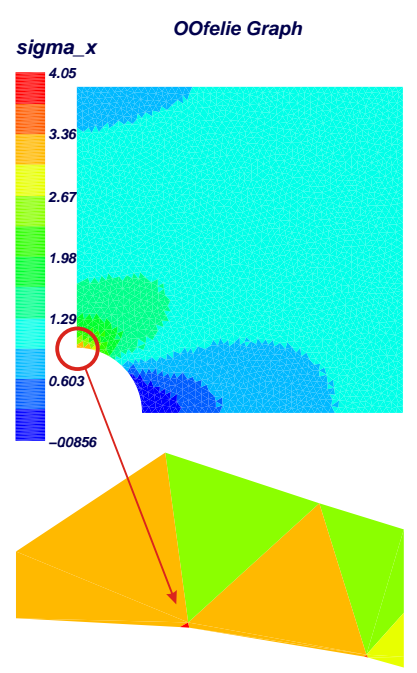

Fig. 13 X-FEM stress over estimation with a small solid element

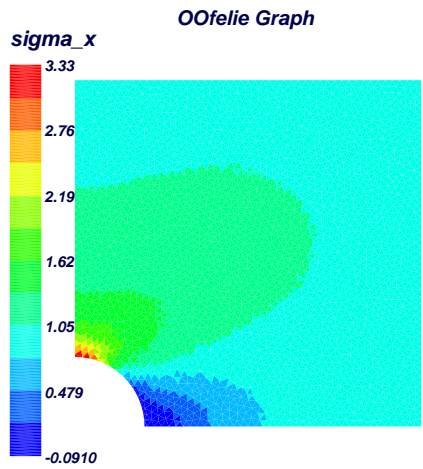

Fig. 14 X-FEM stress estimation without small solid elements

- Compute nodal stresses using a smoothing procedure as in Finite Element error estimation.

The first and second ones sound a bit tricky, while the fourth solution is obviously the best one. Because this solution was not available in our computer code, we preferred here the third solution because of it simplicity since it is just a matter of post-processing the results.

\section{The Level Set Description}

The low performances generally obtained with the shape optimization is directly related to the fact that no deep boundary or topological changes such as creation or fusion of holes is allowed with an explicit parametric CAD representation. This restriction is overcome when using an implicit representation based on the level Set description.

The Level Set method, introduced by Osher and Sethian in Ref. (23), is a numerical technique first developed to track moving interfaces in physics problems. It is based upon the 
idea of implicitly representing the interfaces as a Level Set curve of a higher dimension function $\psi(\mathbf{x}, t)$. The boundaries of the structure are then conventionally represented by the zero level i.e. $\psi(\mathbf{x}, t)=0$ of this function $\psi$, whereas the filled region is attached to the positive part of the $\psi$ function (see Fig. 15).

If one knows also the parametric expression of the curve $\gamma$, a corresponding Level Set can be easily constructed using the signed distance function to the curve $\gamma$ :

$\psi(\mathbf{x}, t)= \pm \min _{\mathbf{x}_{\gamma} \in \gamma(t)}\left\|\mathbf{x}-\mathbf{x}_{\gamma}\right\|$

Level Sets can be easily combined using Boolean operations via min or max operators. This allows to create new entities and to model complex geometries.

\subsection{Level Set and X-FEM}

Applied to the X-FEM framework, the Level Set is defined on the structural mesh and a geometrical degree of freedom representing its Level Set function value is associated at each element node. Inside the elements, the Level Set is interpolated with the shape function used for a finite elements approximation:

$\psi(\mathbf{x}, t)=\sum_{i} \psi_{i} N_{i}(\mathbf{x})$

The sign of $\psi$ is then used to know whether or not a node lies in the material domain. It is also used to give a direct access to the type of the elements (solid, void or cut) and to the cut edges of the finite elements:

- a triangle with 3 positive (negative) values of the Level Set if filled with material (void);

- a triangle which has both positive and negative values is cut.

$\psi(\mathbf{x}, t)>0 \Leftrightarrow$ Solid
$\psi(\mathbf{x}, t)<0 \Leftrightarrow$ Void

Besides these advantages, the Level Set can also present some drawbacks. For instance in optimization, the discretization approximation of eqn. (9) interpolates linearly the Level Set when first order finite element are used. As a consequence, the representation of the boundaries can overestimate or underestimate the surface (volume) of the structure. Hence, the estimation of the surface depends on the number of cut elements, on the position of the inteface inside the element and of course on the mesh refinement. This error can causes some "zigzagging" problems during optimization if one uses a very coarse mesh and a constraint or objective function related to the area (volume) as pointed out in Ref. (33).

\section{Formulation of optimization problem}

The formulation of the considered optimization problem is similar to a shape optimization problem, but its solution is

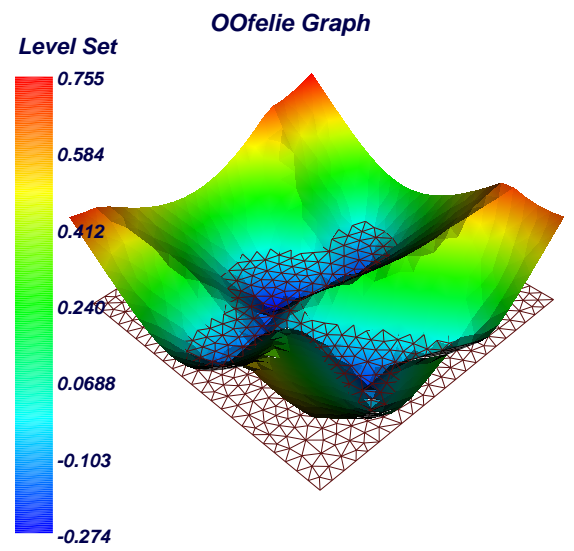

(a) Level Set representation of NURBS curve

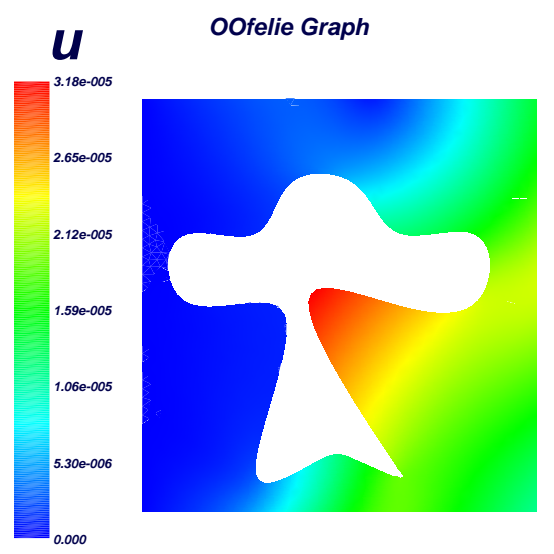

(b) Displacement of the X-FEM model

Fig. 15 Geometric representation and X-FEM displacement result

greatly simplified thanks to the use of the X-FEM and Level Set description as no velocity field and mesh perturbation are needed.

The geometry and the material domains are specified using Level Sets description of the boundaries. The positive part of the Level Set represents material domain while the negative part is the void. To describe the structural geometry the user has a library of basic geometric features (in Level Sets) that can be combined to create almost any structural geometry. The library geometric features are circles, ellipses and all polygons. The design variables are chosen among the geometric parameters of these features.

The optimization problem aims at finding the best shape for minimizing a given objective function while satisfying mechanical and geometrical design restrictions. The mechanical constraints can either be global responses (e.g. compliance, volume) or local ones such as displacements or stress constraints.

The number of design variables is generally small as in shape optimization. However the number of constraints may be large if many local stress restrictions (e.g. stress con- 
straints) are considered. Nonetheless, large scale problems as in topology optimization are avoided.

The design problem is cast as a general constrained optimization problem:

$$
\begin{array}{cl}
\min & g_{0}(\mathbf{x}) \\
\mathbf{x} & \\
\text { s.t.: } & g_{j}(\mathbf{x}) \leq g_{j}^{\max } j=1 \ldots m \\
& \underline{x}_{i} \leq x_{i} \leq \bar{x}_{i} \quad i=1 \ldots n
\end{array}
$$

The solution to this problem is obtained using the so-called sequential convex programming. At each iteration, the $\mathrm{X}$ FEM analysis problem is solved and a sensitivity analysis is performed. The solution of the optimization problem is then found by using a CONvex LINearization approximation scheme of each constraint functions (see (15)). The solution becomes the new design and the procedure is repeated until convergence.

Because of the X-FEM characteristics, the geometry has not to coincide with the mesh and the shape optimization problem is carried out on a fixed mesh. One works here in an eulerian approach and not in a lagrangian approach. This circumvents the mesh perturbation problems of classical shape optimization. Sensitivity analysis can be performed without considering a velocity field. The present formulation is then, up to a certain point, simpler. However, some technical difficulties can be encountered if a finite difference or a semianalytical scheme is used for sensitivity analysis. Basically, the problem is that the perturbation must not change the number of degrees of freedom of the X-FEM stiffness matrix while a finite perturbation of the Level Set could introduce new cut element and new nodes into the formulation.

The Level Set approach is very convenient to modify the geometry because the Level Sets (and so the holes) can penetrate each other or disappear. Creation of new holes is more problematic since it leads to a non smooth problem. Topological derivatives have then to be used for a rigorous treatment of the problem (see ref. (21) for instance). This capability has not been implemented in this study.

\section{The sensitivity analysis method}

Similarly to classical shape optimization, the sensitivity analysis of the various responses (such as compliance, displacements, stresses, ...) is carried out using a semi-analytic approach. The derivatives of stiffness matrix (K) and load vectors (f) are calculated by finite differences with respect to a small perturbation $\delta x$ of Level Set parameters:

$$
\begin{aligned}
\frac{\partial \mathbf{K}}{\partial x} & \simeq \frac{\mathbf{K}(x+\delta x)-\mathbf{K}(x)}{\delta x} \\
\frac{\partial \mathbf{f}}{\partial x} & \simeq \frac{\mathbf{f}(x+\delta x)-\mathbf{f}(x)}{\delta x}
\end{aligned}
$$

These derivatives are then used to compute the sensitivity of the various objective functions. For the compliance $C$, the sensitivity (in the case of invariant loading forces) is then given by:

$\frac{\partial C}{\partial x}=-\frac{1}{2} \mathbf{u}^{T} \frac{\partial \mathbf{K}}{\partial x} \mathbf{u}$

Now, if the objective function or constraint involves the stresses of the problem, the sensitivity of this response is needed. Two basic methods are available to get the derivative of the stresses. The first one, which has the drawback of forcing us to know explicitly the tension matrix, consists in deriving the expression of the stresses $(\sigma)$ in all the elements:

$\sigma=\mathbf{T}_{\mathbf{j}} \mathbf{u}_{\mathbf{j}}=\mathbf{H B}_{\mathbf{j}} \mathbf{u}$

where $\mathbf{H}$ is the Hooke's matrix, $\mathbf{B}_{\mathbf{j}}$ the matrix of the derived shape functions of the element $j$ and $\mathbf{T}_{\mathbf{j}}$ the tension matrix of element $j$. The second method is based on the computation of the stresses related to the perturbed state $x+\delta x$ by using the expression of the displacement sensitivities:

$$
\begin{aligned}
\sigma(x) & =\mathbf{H B}_{\mathbf{j}} \mathbf{u}(x) \\
\mathbf{u}(x+\delta x) & \simeq \mathbf{u}(\mathbf{x})+\frac{\partial \mathbf{u}}{\partial x} \delta x \\
\sigma(x+\delta x) & \simeq \mathbf{H B}_{\mathbf{j}} \mathbf{u}(x+\delta x) \\
\frac{\partial \sigma}{\partial x} & \simeq \frac{\sigma(x+d x)-\sigma(x)}{\partial x}
\end{aligned}
$$

This procedure reduces the sensitivity of the stresses as a function of the displacement derivative. In the present paper, the implementation is performed in an industrial code OOFELIE for which the knowledge of tension matrix $\mathbf{T}_{\mathbf{j}}$ is not available for any element. Moreover for the maintenance of industrial code it is often preferred to assume that sensitivity analysis is made less dependent upon element implementation. Therefore, the second method has been chosen and implemented.

\section{OOfelie Graph}

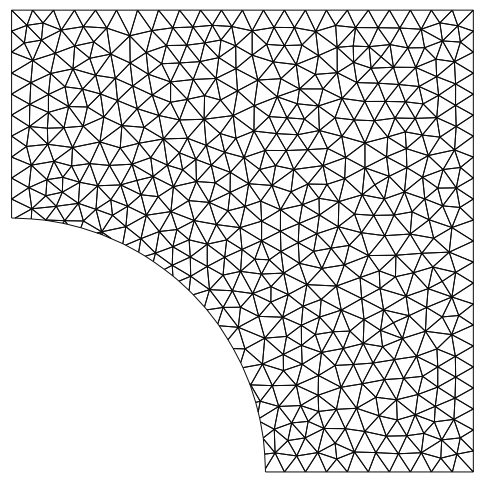

Fig. 16 X-FEM Mesh for sensitivity analysis validation 
The quarter plate with an elliptical hole and non uniform bi-axial loading (Fig. 16) serves as a tool for the validation of the approximated semi-analytical sensitivity analysis that has been used. Table 1 gives the sensitivities of the maximum stress calculated by finite differences and semianalytical approach for different combinations of the design variables $a$ and $b$, the major and minor axes of the elliptical hole. The results were obtained with a relative perturbation of the design variables of $\delta=10^{-4}$. The table presents the quality of the proposed semi-analytical approximation.

Table 1 Validation of semi-analytical sensitivity analysis approximation.

\begin{tabular}{llll}
\hline Variable & Finite difference & Semi-analytical & Relative error \\
\hline$a=0.41$ & -213.2172401 & -213.2279160 & $0.005 \%$ \\
$b=0.41$ & 2075.6012932 & 2074.0930198 & $0.073 \%$ \\
\hline$a=0.55$ & -51.9288917 & -51.888882944695 & $0.077 \%$ \\
$b=0.55$ & 5019.8392594 & 5003.5389826985 & $0.32 \%$ \\
\hline
\end{tabular}

In the classical shape optimization, the computing complexity of the stiffness matrix sensitivity is due to the modifications of the mesh associated to the perturbation $\delta x$ and to the velocity field calculation. In the present X-FEM based approach, one has not to deal with the mesh perturbations as one works on a fixed grid. However, this method exhibits a different drawback with respect to the general shape optimization as, with X-FEM, the number of elements may change. Therefore, when Level Set is perturbed in order to compute the sensitivity, there is a possibility that previously empty element become partly filled with material. As a consequence, the number of degrees of freedom change and the dimension of the stiffness matrix is modified between the Level Set perturbation which forbid to compute the stiffness matrix derivative by finite difference (eqn. 12). The strategy that is implemented presently (see Ref. (33) and (14) for details) to circumvent the difficulty is the following. As one has only the displacement $\left(u_{i}\right)$ for the elements that are present in the reference configuration, only these elements are taken into account while the contributions coming from the new partly filled elements are ignored. Hence, no new elements are introduced and the size of the stiffness matrix remains unchanged. Of course, the ultimate solution to the problem should resort to a fully analytical sensitivity of the stiffness matrix. However, this would be rather restrictive for industrial applications.

\section{Numerical applications}

The X-FEM method for the modeling of material-void discontinuity and its Level Set description have been implemented in an object oriented $(\mathrm{C}++)$ multiphysics finite element code, OOFELIE that is commercialized by Open Engineering (information available on the web site (22)).
In this software, various mechanical responses can be chosen as objective functions and design restrictions that is: compliance and potential energy, all stress components and Von Mises equivalent stress, displacements and geometric results. Presently, the implementation of the X-FEM method is only available in 2-D with a library of both first degree quadrangle and triangle elements. The Level Set description can be defined in different ways. They can be constructed classically from functions (circle, quadrangles, ellipses, ...) or from a set of points which are interpolated by a NURBS curve. The CONLIN optimizer by $\mathrm{C}$. Fleury described in Ref. (15) has been coupled in the OOFELIE environment to realize the numerical applications.

\subsection{The 2D-filet in tension}

The numerical applications are inspired by the famous problems of stress concentration factors evaluation as in (29). Pedersen in Ref.(27) has stated this problem as an optimization problem. The problem consist in studying the effect of the connection zones in the following 2D structure and reducing the stress concentration factor.

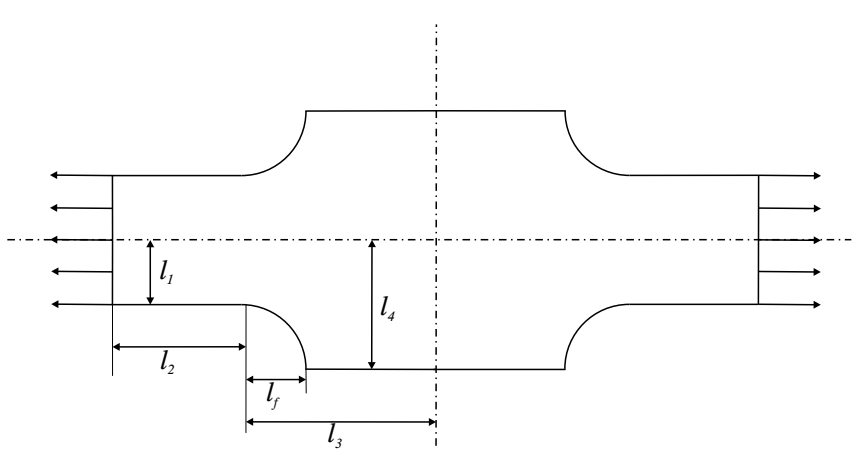

Fig. 17 2D filet model in tension

The following geometry parameters are fixed to the values:

- $l_{1}=30 \mathrm{~mm}, l_{4}=60 \mathrm{~mm}, l_{2}=30 \mathrm{~mm}, l_{3}=90 \mathrm{~mm}$;

- $l_{f}=30 \mathrm{~mm}$ for section 6.1.1 and 6.1.2 and is a variable for the section 6.1.3.

The plane stress state is assumed and a uni-axial stress field is applied $\sigma_{x}=1$. The material properties associated are: Young's modulus $E=1 \mathrm{~N} / \mathrm{m}^{2}$, Poisson's ration $v=0.3$. Due to symmetries, only a quarter part is studied.

\subsubsection{Super circular connection}

As P. Pedersen pointed out in Ref.(27), a circular connection to a straight domain is not the best solution and it is better to replace it by a super-circular connection as defined by:

$x_{1}^{\eta}+x_{2}^{\eta}=r$ 


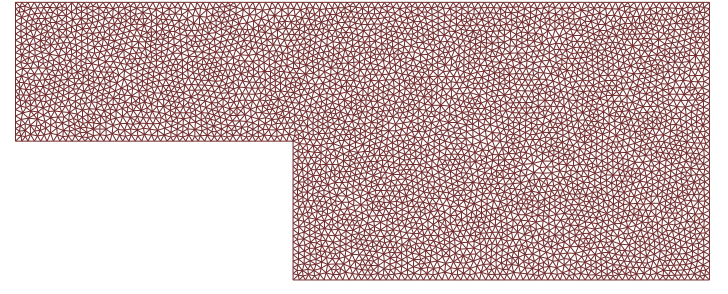

(a) Mesh used for all the test cases

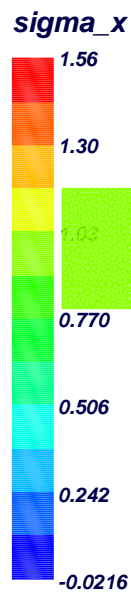

(b) Initial configuration: $\eta=2, \sigma_{\max }=1.563$

Fig. 18 Mesh and X-FEM analysis

Since a parameter is introduced, the goal of the optimization problem is to find the configuration presenting the minimum stress concentration:

$\min \max _{i} \sigma_{i_{x}}^{2}$

where $i$ is the index of the finite elements. In practice, a multi objective approach is used by taking into account only the most critical elements, i.e. the top $10 \%$ of the most stressed finite elements. No other constraint is prescribed in the problem except the side-constraint over the variable $\eta$ which restricts $\eta$ to the domain $2 \leq \eta \leq 4$. The initial configuration, with $\eta=2$, presents a stress concentration $\sigma^{\max } / \sigma^{\infty}=$ 1.56253 with the mesh given in Fig. 18 (8000 degrees of freedom). The optimum, obtained after ten iterations, provide a rather limited improvement as the stress concentration is reduced to the value of $\sigma^{\max } / \sigma^{\infty}=1.52612$ (gain of $2.75 \%$ ) and an optimum value of $\eta=2.0935$ (Fig. 19).

\subsubsection{Generalized super circular connection}

The previous test case shows that only a minor improvement can be obtained by using a super circle in comparison to a normal circle. Therefore, we replace the super circle filet with a generalized super circle defined as:

$x_{1}^{\eta}+x_{2}^{\xi}=r$

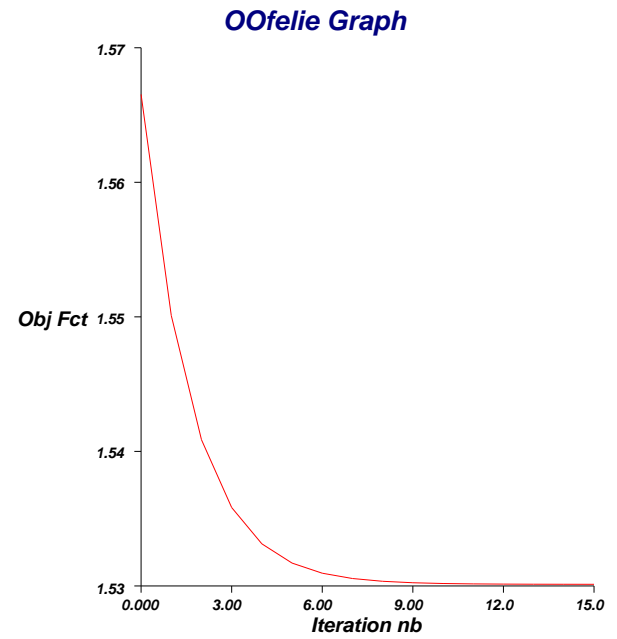

(a) Evolution of the maximum stress

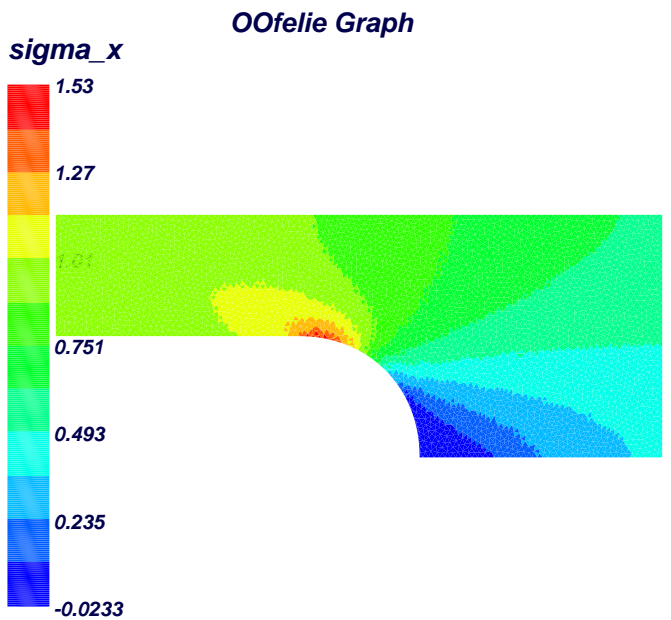

(b) Final configuration: $\eta=2.09, \sigma_{\max }=1.526$

Fig. 19 Optimization with a super circle

The problem is defined as the previous one, however it handles now two variables, $\xi$ and $\eta$ which are constrained between the values 2 and 4 . As it can be remarked, the additional exponent allows a deepest modification of the filet curvature which leads to a significant reduce of the stress concentration of $6.7 \%$ (Fig. 20).

\subsubsection{Generalized super ellipse connection}

This last test case shows the important effect of the filet length on the stress concentration. A generalized super ellipse is used to define the boundary of the filet:

$\left|\frac{x_{1}}{a}\right|^{\eta}+\left|\frac{x_{2}}{b}\right|^{\xi}=r$

With an unlimited filet length this stress concentration could be avoided. Hence, the optimization variable $a$ is constrained 


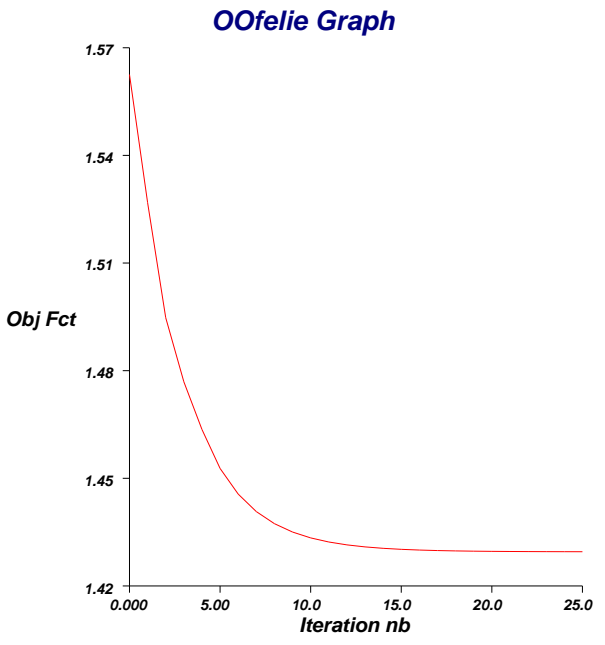

(a) Evolution of the maximum stress

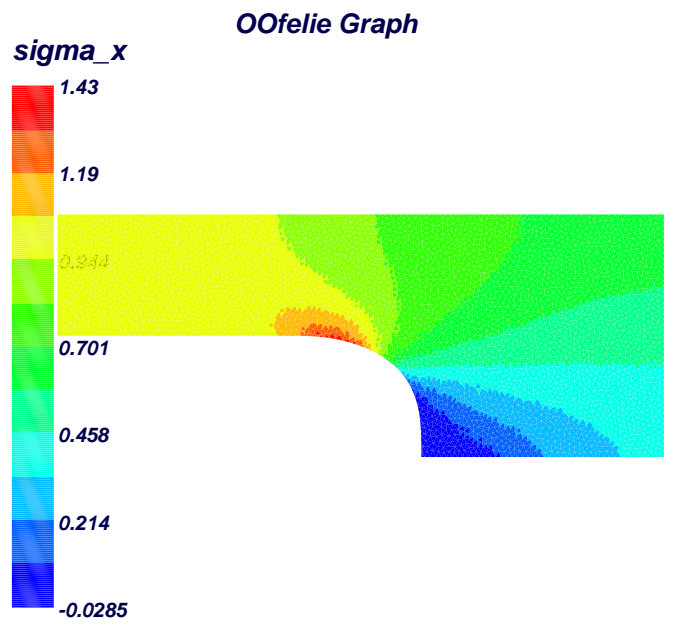

(b) Final configuration: $\xi=3.259, \eta=2.09, \sigma_{\max }=1.42938$

Fig. 20 Optimization with a generalized super circle

between the values 20 and 60 (initial value at 30). The other variables are constrained between $2 \leq \xi \leq 4$ and $2 \leq \eta \leq 4$.

As expected, the optimization reaches the same $\xi$ and $\eta$ obtained with the previous application and maximizes the value of the parameter $a$ representing the filet length. The stress concentration has been reduced by $22 \%$ with respect to the previous case and nearly $30 \%$ to the initial circular filet (Fig. 21).

\section{CONCLUSIONS}

This work has presented an novel approach for shape optimization based on the Level Set description and the X-FEM for the structural optimization. This new approach takes place between shape and topology optimization as it pos-

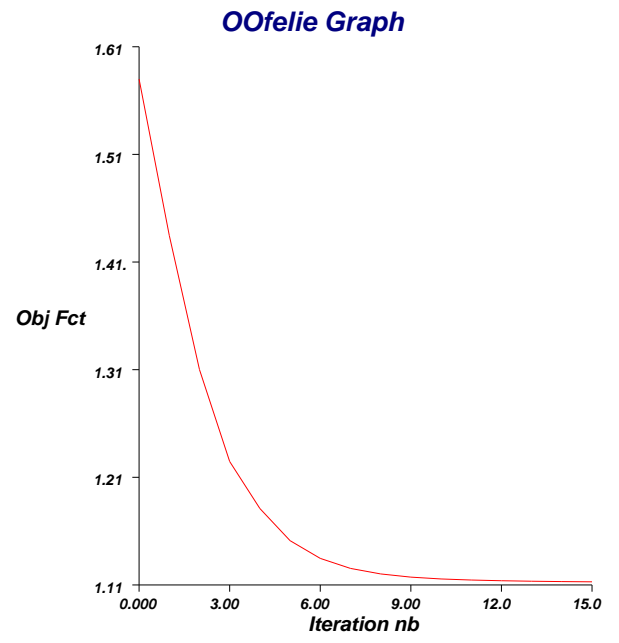

(a) Evolution of the maximum stress



(b) Final configuration: $\quad \xi=3.259, \quad \eta=2.09, \quad a=60$, $\sigma_{\max }=1.11809$

Fig. 21 Optimization with a generalized super ellipse

sesses the same characteristics of both ones. The X-FEM method has shown to be very useful as it easily takes advantage of the fixed mesh work approach of topology optimization whereas the smooth curve description from the shape optimization is preserved. Moreover, void is not approximated as a smooth material in opposition to the SIMP method. Contrary to shape optimization, no remeshing process is needed in our applications, and only one mesh has been created and used for all the test cases.

A semi-analytic sensitivity analysis with X-FEM and Level Set for various responses have been developed and validated. One issue that has been investigated is the quality of the X-FEM approximation for stresses. The source of the problems are the small elements that have only a very low fraction of their surface filled with solids. These elements 
leads to large errors in the stress estimation. The estimation for these elements should not be considered in a rough way, but rather require special post processing.

The novel shape optimization approach based on the XFEM and Level Set description has been illustrated with the minimization of the stress concentration of a 2D mechanical structure. It gives encouraging results to study large scale industrial problems, especially 3-D problems. Other analyses problems will also be investigated in the future as dynamic problems and multiphysics (electro-mechanical) problems.

\section{Acknowledgments}

The authors gratefully acknowledge the support of project ARC MEMS, Action de recherche concertée 03/08-298 funded by the Communaute Francaise de Belgique.

\section{References}

1. Allaire G. Jouve F. and Toader A.M. (2004) Structural optimization using sensitivity analysis and a level-set method. Journal of Computational Physics. Vol. 194, Issue 1, pp 363-393.

2. Allaire G., Jouve F. and Maillot H. (2004) Topology optimization for minimum stress design with the homogenization method. Structural and Multidisciplinary Optimization Vol. 28, No 2-3, 87-98.

3. Bendsøe M. P. and Kikuchi N. (1988). Generating Optimal Topologies in Structural Design Using a Homogenization Method, Computer Methods in Applied Mechanics and Engineering, 1988, 71, 197-224.

4. Belytschko T., Moës N., Usui S., Parimi C.(2001) Arbitrary discontinuities in finite elements. Int. J. Num. Meth. in Engng 50: 9931013.

5. Belytschko. T. Parimi C. Moes N., Sukumar N. and Usui. S. (2003). Structured extended finite element methods for solids defined by implicit surfaces. Int. J. Numer. Meth. in Engng 2003; 56 : 609-635.

6. Belytschko T., Xiao S. and Parimi C. (2003). Topology optimization with implicit functions and regularization. Int. J. Num. Meth. in Engng 2003; 57 : 1177-1196.

7. Bennet J.A. and Botkin E. (1985) Structural Shape Optimization with Geometric Description and Adaptive Mesh Refinement. AIAA Journal Vol 23 No 3, 458-464.

8. Braibant V. and Fleury C. (1984) Shape optimal design using Bsplines. Comput. Meths Appl. Mech Engrg. Vol 44 No 3, 247-267.

9. Chessa J. and Belytschko T.(2003) The Extended Finite Element Method for Two-Phase Fluids. ASME Journal of Applied Mechanics, 70(1): 10-17.

10. Dankova . and Haslinger J. (1996) Numerical realization of a fictitious domain approach used in shape optimization. Part I Distributed controls. Applications of Mathematics 1996, 41(2): 123-147.

11. Daux C., Moës N., Dolbow J., Sukumar N., Belytschko T.(2000) Arbitrary discontinuities in finite elements. Int. J. Num. Meth. in Engng 48: 1741-1760.

12. Ding Y. (1986) Shape optimization of structures: a literature survey. Computers and Structures. Vol 24 No 4, 985 - 1004.

13. Duysinx P. and Bendsøe M.P. (1988) Control of local stresses in topology optimization of continuum structures, International Journal for Numerical Methods in Engineering, 1998, 43, 1453-1478.

14. Duysinx P., Van Miegroet L., Jacobs T. and Fleury C. (2006) Generalized Shape Optimization Using X-FEM and Level Set Methods, IUTAM Symposium on Topological Desgin, Optimization of Structures, Machines and Materials, Bendsøe M. P., Olhoff N. and Sigmund O. Eds. 2006, 23-32.
15. Fleury C. (1989) CONLIN: an efficient dual optimizer based on convex approximation concepts. Structural Optimization. Vol. 1, pp 81-89.

16. Guétari Y., Le Corre S., Moës N. (2005) Étude des possibilités de la méthode X-FEM pour la simulation numérique de la coupe. Mécanique \& Industries 6: 315-319.

17. Haftka R.T. and Grandhi R.V. (1986). Structural shape optimization - A survey. Computer Methods in Applied Mchanics and Engineering Vol 57, 91-106.

18. Kim H., Querin O.M., Steven G.P., Xie Y.M. (2002). Improving efficiency of evolutionary structural optimization by implementing fixed grid mesh. Structural and Multidisciplinary Optimization Vol 24, No 6, 441-448.

19. Moes N., Dolbow J., Sukumar N. and Belytschko T. (1999) A finite element method for crack growth without remeshing. International journal for numerical methods in engineering 1999, no. 46, 131-150.

20. Norato J., Haber R., Tortorelli D. and Bendsøe M.P. (2004) A geometry projection method for shape optimization. Int. J. Num. Meth. in Engng 1999, 60(14): 2289-2312.

21. Novotny A.A., Feijoo R.A., Taroco E. and Padra C. (2003) Topological Sensitivity Analysis. Comput. Methods Appl. Mech. Engrg. 2003, 192, 803-829

22. OOFELIE, an Object Oriented Finite Element Code Led by Interactive Execution. Internet resources: www.open-engineering.com.

23. Osher, S. and Sethian, J. A (1988) Fronts Propagating with Curvature-Dependent Speed: Algorithms Based on Hamilton-Jacobi Formulations. Journal of Computational Physics 1988, Vol. 79, 12 49.

24. Pedersen P. and Laursen C.L. (1983) Design for Minimum Stress Concentration by Finite Element and Linear Programming. J. structural Mech. Vol 10 No 4, 375-391.

25. Pedersen P. (1991). Optimal shape design with anisotropic materials. NATO/DFG ASI Proceeding of vol. 1 Optimization of Large Structural Systems (Dir. G. Rozvany), Berchtesgaden, Germany, Sept. 23 - Oct. 41991.

26. Pedersen P. (2000) On optimal shapes in materials and structures. Structural Optimization Vol 19, No 3, 169-182.

27. Pedersen P. (2003) Optimal Designs - Structures and Materials Problems and Tools -, May 28, 2003.ISBN 87-90416-06-6

28. Pereira J., Fancello E. and Barcellos D.S. (2004) Topology optimization of continuum structures with material failure constraints. Structural and Multidisciplinary Optimization. Vol. 26, No 1-2, 5066.

29. Peterson (1997) Stress Concentration factors, Second edition, John Wiley and Sons, 1997.

30. Rozvany G.I.N. (1996). Some Shortcomings in Michell's Truss Theory. Structural Optimization, Vol.12, 244-250.

31. Sukumar N., Chopp D. L., Moës N. and Belytschko T. (2001) Modelling holes and inclusions by Level Set in the extended finite element method. Comput. Methods Appl. Mech. Engrg. 2001, 190, pp 6183-6200

32. Van Miegroet L., Moes N., Fleury C. and Duysinx P. (2005). Generalized shape optimization based on the Level Set method. Proceedings of the 6th World Congress of Structural and Multidisciplinary Optimization (J. Herskowitz ed.), Rio de Janeiro, Brazil, May 30 June 32005 .

33. Van Miegroet L., Lemaire E., Jacobs T., Dusyinx P. (2006). Stress constrained optimization using X-FEM and Level Set description. Proceedings of the 3rd European Conference on Computational Mechanics. Shape and Topological Sensitivity Analysis: Theory and Applications, Lisbon, Portugal, June 5 - 92006.

34. Wang M., Wang X. and Guo D. (2003). A Level Set method structural topology optimization. Comp. Methods in Appl. Mech Engng. 2003; 191:227-246. 